\title{
MICROWAVE SCATTERING PROFILES OF A RICE SAMPLE BY MEANS OF POLARIZATION COHERENCE TOMOGRAPHY
}

\author{
Maria-Jose Sanjuan, Juan M. Lopez-Sanchez, J. David Ballester-Berman \\ Signals, Systems and Telecommunication (SST) Group \\ DFISTS, University of Alacant, P.O. Box 99, E-03080 Alacant/Alicante, Spain \\ Phone / Fax: +34965909597 / 9750 , \\ E-mail: masanfe4@hotmail.com,juanma.lopez@ua.es, davidb@dfists.ua.es
}

\begin{abstract}
In this work we have applied the polarization coherence tomography (PCT) to indoor data acquired on a rice sample. Different baselines, frequency bands and polarimetric channels have been used in order to analyze the potential of this technique for retrieving information about the vertical structure of such a specific crop and, additionally, these observations have been also used for studying the validity of the RVoG and OVoG models. Results obtained show that the PCT profiles exhibit no dependence on polarization, which is in agreement with the clustering effect observed on PolInSAR observables, i.e. coherences for different polarimetric channels are located very close one to another. In the dual-baseline case, the best profile reconstruction is obtained for the highest baseline ratio available $\left(0.25^{\circ}\right.$ and $1^{\circ}$ baselines $)$ when the condition number of the inversion problem is improved by filtering out the fifth coefficient of the Legendre-Fourier expansion. Differences on the reconstructed profiles between linear and Pauli basis are negligible. The application of the triple- and four-baseline approaches without filtering out the last terms of the expansion leads to unstable results, whereas the filtered cases reveal that the contribution of a fourth baseline is null.
\end{abstract}

Index Terms - Polarimetric SAR interferometry, vegetation, agriculture, tomography, indoor experiments.

\section{INTRODUCTION}

A new imaging technique, called Polarization Coherence Tomography (PCT), has been recently formulated and demonstrated in two scenarios: a Scots Pine forested area at L-band, and a maize sample observed in laboratory conditions $[1,2]$. This method makes use of PolInSAR observables (complex interferometric coherences at different polarizations) in order to reconstruct the vertical profile of microwave scattering

This work has been supported by the Spanish Ministry of Education and Science (MEC) and EU FEDER under Projects TEC2005-06863-C02-02 and HA2007-0075. from the vegetated scene. This task is carried out by expanding the vertical profiles which contribute to the coherences by means of Fourier-Legendre polynomials. Vegetation height and the ground vertical position are used as input parameters when they are known. Single-baseline and dual-baseline cases have been already illustrated in the literature.

The applications of this approach are manifold. In the field of microwave remote sensing for agriculture, this approach offers an important potential to recover information about the scattering processes that take place inside the volume, specially concerning the assumptions adopted for describing the scattering mechanisms along the vertical structure. Hence, this new technique could be used for validating the assumptions of the direct models and for interpreting the results provided by existing retrieval approaches.

The analysis presented in this paper is focused on a sample of rice, which is an important crop from economic and social points of view. The PCT technique has been applied to wide-band fully polarimetric data acquired in laboratory conditions with different incidence angles, thus providing a multi-baseline dataset.

The first objective of this work is to assess the dependence of the retrieved profiles upon the polarimetric channel, and linking this dependence to the expected behavior based on the scattering physics. In second place, the validity of a widely used direct model (i.e. RVoG and $\mathrm{OVoG}$ ) for characterizing the response from rice has been studied. Finally, the contribution of additional baselines (more than two) has been also tested.

\section{PRINCIPLE OF THE PCT}

Starting from measurements of the complex interferometric coherence $\gamma$, the aim of the PCT is to derive a vertical profile or vertical structure function $f(z)$ which represents the vertical variation of microwave scattering at a point in the twodimensional radar image. The reconstruction of the function $f(z)$ at each point in the image is then termed coherence to- 
mography. The linear inversion problem to be solved is defined as:

$$
\vec{a}=[F]^{-1} \vec{b}
$$

where $\vec{a}$ correspond to the unknown coefficients of the Legendre-Fourier expansion, matrix $[F]$ contains functions $f_{n}$ obtained by means of the Legendre polynomials, and $\vec{b}$ are the observables (i.e. the coherences).

As an extension of the the formulation originally presented in [1, 2], expressions for triple- and four-baseline tomography can be easily derived in order to study the effect of increasing the number of coefficients for retrieving the vertical structure function $f(z)$. Ideally, this would lead to a higher resolution but, as we will show later, the inversion problem becomes worse conditioned. The new additional functions $f_{n}$ appearing in matrix $[F]$, required for reconstructing the vertical profiles for triple- and four-baselines approaches, are given in the following:

$$
\begin{aligned}
f_{5} & =j\left[\frac{-2 k_{v}^{4}+210 k_{v}^{2}-1890}{k_{v}^{5}} \cos k_{v}+\right. \\
& \left.+\frac{30 k_{v}^{4}-840 k_{v}^{2}+1890}{k_{v}^{6}} \sin k_{v}\right] \\
f_{6} & =\frac{42 k_{v}^{4}-2520 k_{v}^{2}+20790}{k_{v}^{6}} \cos k_{v}+ \\
& +\frac{2 k_{v}^{6}-420 k_{v}^{4}+9450 k_{v}^{2}-20790}{k_{v}^{7}} \sin k_{v} \\
f_{7} & =j\left[\frac{-2 k_{v}^{6}+756 k_{v}^{4}-34650 k_{v}^{2}+270270}{k_{v}^{7}} \cos k_{v}+\right. \\
& \left.+\frac{56 k_{v}^{6}-6300 k_{v}^{4}+124740 k_{v}^{2}-270270}{k_{v}^{8}} \sin k_{v}\right] \\
f_{8} & =\frac{72 k_{v}^{6}-13860 k_{v}^{4}+576576 k_{v}^{2}-4054050}{k_{v}^{8}} \cos k_{v}+ \\
+ & \frac{2 k_{v}^{8}-1260 k_{v}^{6}+103950 k_{v}^{4}-1891890 k_{v}^{2}+4054050}{k_{v}^{9}} \sin k_{v}
\end{aligned}
$$

\section{RESULTS}

\subsection{Tomograms for different configurations}

Results obtained for different configurations are discussed in this section. Details of the experimental dataset can be found elsewhere [3]. First, single-baseline tomograms have been computed for linear and Pauli basis. The vertical profiles in the frequency range 5-9 GHz are shown in Fig. 1.a for the $\mathrm{HV}$ basis and $0.75^{\circ}$ angular baseline. All reconstructed profiles are very similar, i.e. they do not show any dependence on polarization, and the maximum scattering is located at the ground level. This behavior is explained because of the short vegetation depth and the strong response from the ground (in this case, from the ground-stem and ground-canopy interactions), which lead to negligible differences in the location of

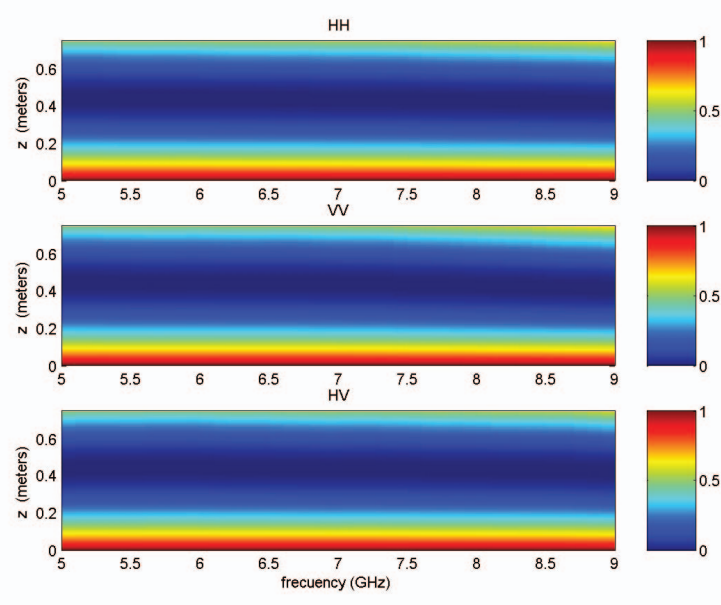

(a) Single-baseline with $B=0.75^{\circ}$
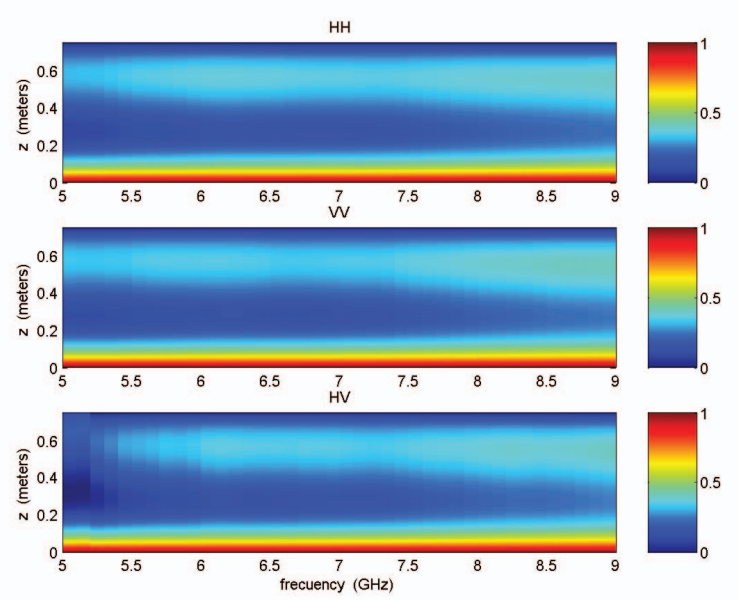

(b) Dual-baseline with $B_{1}=0.25^{\circ}$ and $B_{2}=1^{\circ}$

Fig. 1. Tomograms with the linear HV basis

the coherences on the complex plane. These observations are illustrated in Fig. 3, where the set of HV and Pauli coherences for baselines of $0.25^{\circ}, 0.5^{\circ}, 0.75^{\circ}$ and $1^{\circ}$ are plotted. It is also seen that single-baseline reconstructions yield a second local maximum at the top of the volume. This scattering center matches the general behavior of a homogeneous volume with a non-zero extinction, but the retrieved shape of the profile must not be trusted due to the low resolution of the single-baseline approach, as reported in [1], since only two coefficients of the expansion of $f(z)$ are computed.

The vertical profiles obtained from the cross-polar channel deserve a particular analysis. In this dataset, total backscattering at the $\mathrm{HV}$ channel is always $10 \mathrm{~dB}$ below the copolar channels, hence demonstrating the low density of the canopy (with randomly oriented leaves) of the rice sample, which is the main origin of HV scattering. However, 

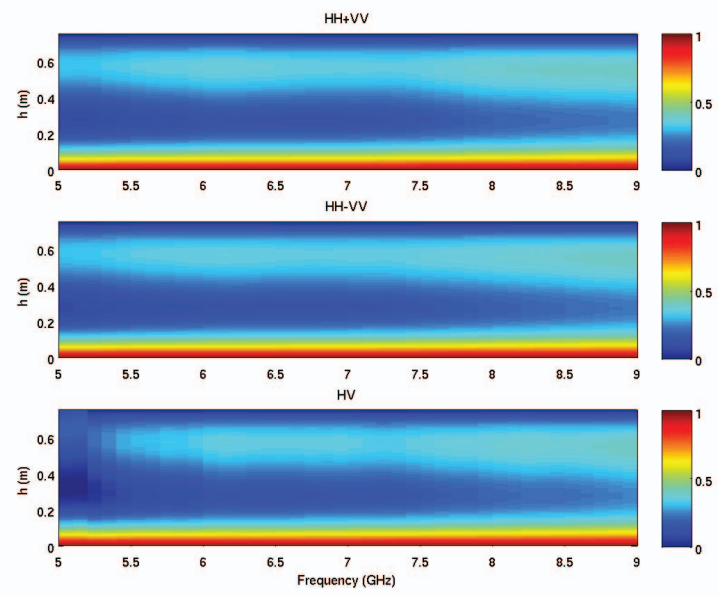

Fig. 2. Dual-baseline tomogram with the Pauli basis: $B_{1}=0.25^{\circ}$ and $B_{2}=1^{\circ}$

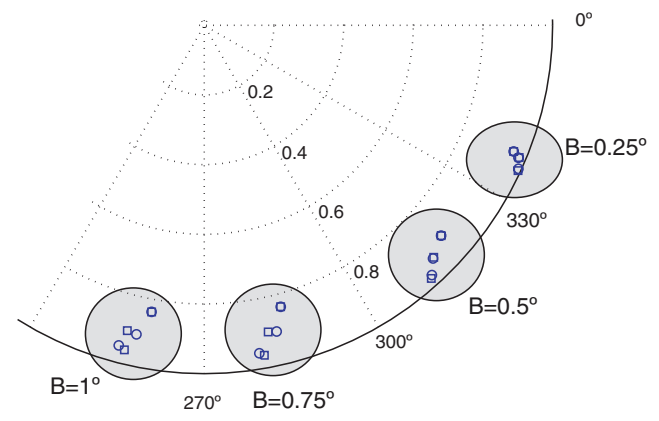

Fig. 3. Coherences in the Pauli and linear basis on the complex plane for $0.25^{\circ}$ to $1^{\circ}$ baselines at $7 \mathrm{GHz}$

it must be considered that PCT profiles are extracted from interferometric coherences, which are actually sensitive to the phase centers distribution and defined by normalizing the total power. Consequently, the assumption of a doublebounce contribution between canopy and flooded soil for the HV channel justifies the retrieved profiles, since the corresponding phase center is located at the ground interface.

The dual-baseline vertical profiles for the linear (HV) basis are plotted in Fig. 1.b. The use of $0.25^{\circ}$ and $1^{\circ}$ baselines, i.e. the maximum baseline ratio available, yields the best performance in agreement with the expected response of such a target. Note again that the response of ground dominates, whereas the backscattering from the canopy is weaker and localized close to the upper part of the plant. This behavior is slightly more noticeable for higher frequencies. The dualbaseline profiles corresponding to the Pauli basis are shown in Fig. 2 and are very similar to those obtained with the linear basis.

Results shown in Figs. 1.b and 2 have been obtained by filtering out the last coefficient of the Legendre-Fourier expansion, as explained in [2]. When no filtering is applied on the
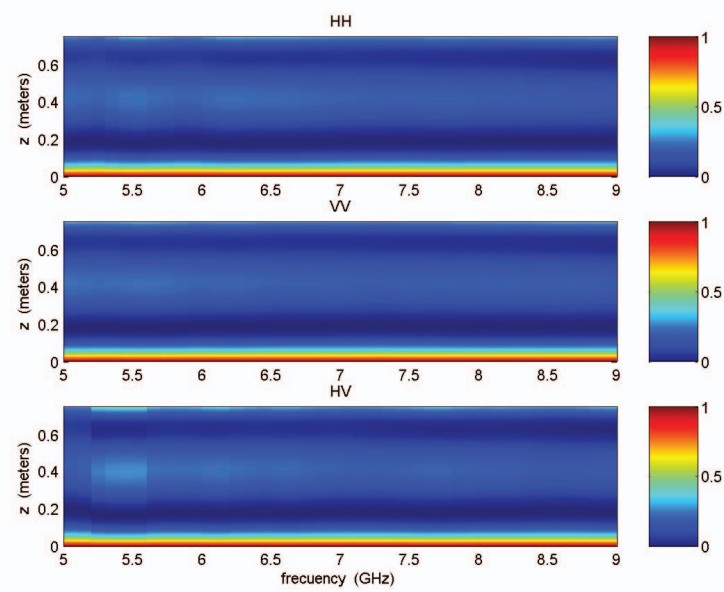

Fig. 4. Triple-baseline tomogram with the HV basis: $B_{1}=0.5^{\circ}$, $B_{2}=0.75^{\circ}$, and $B_{3}=1^{\circ}$

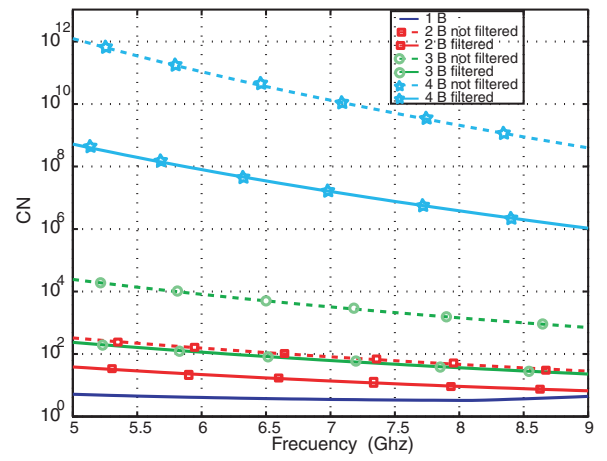

Fig. 5. Condition number as a function of frequency for multibaseline PCT

coefficients computation, the retrieved profiles become unstable and useless. In addition, for the dual-baseline approach, other lower baseline ratios have been also used but the results are not so consistent with the scattering physics. Therefore, this result is in agreement with the comments in [1] concerning the conditioning of the inversion problem for the dualbaseline approach.

Approaches with three and four baselines have been also tested, but results obtained do not yield any improvement on the expected vertical profiles, and even they get worse when compared to the dual-baseline case.

Tomograms corresponding to the triple-baseline case are plotted for the linear basis in Fig. 4. In this case, the two last coefficients of the expansion were filtered out for a better conditioning of the inversion, but the condition number is always worse than in the dual-baseline case after filtering (see Fig. 5), thus limiting the fidelity of the profiles. These results, which are similar to those obtained for the four-baseline approach, show that the maximum is again at the ground level but the scattering from the volume is now divided into a layer around 

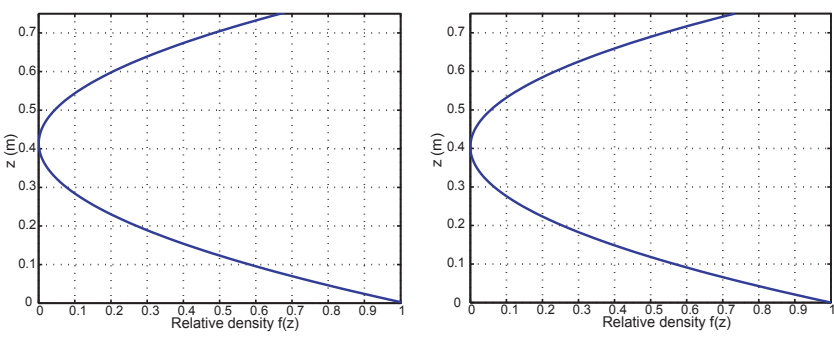

Fig. 6. Single-baseline $x$-pol vertical profile from the (left) RVoG and (right) OVoG models. Parameters: $\theta_{0}=44^{\circ}, f=7 \mathrm{GHz}$, $B_{1}=0.25^{\circ}, B_{2}=1^{\circ}, \mu=3 \mathrm{~dB}, \sigma=3 \mathrm{~dB} / \mathrm{m}$ for RVoG, $\sigma_{a}=3.5 \mathrm{~dB} / \mathrm{m}$ and $\sigma_{b}=2.75 \mathrm{~dB} / \mathrm{m}$ for OVoG, $z_{0}=-0.24 \mathrm{~m}$

the center of the volume and a second smaller maximum at the top.

\subsection{Assessment of the RVoG and OVoG model}

In order to assess the assumptions of the $\mathrm{RVoG}$ and $\mathrm{OVoG}$ models $[4,6,5]$, we have analyzed the profiles derived from the theoretical coherences provided by these models.

To adapt the analysis to the rice, we have used input parameters values provided by estimates from the inversion of the RVoG and OVoG models for the same rice sample [3]. Then, the resulting theoretical coherences are used as inputs for the PCT technique for recovering the vertical profiles associated with this target parameters. Results for the crosspolar channel at $7 \mathrm{GHz}$ are plotted in Figures 6 and 7 for single- and dual-baseline cases, respectively.

In general, the variation of the relative density function agrees with the overall behavior explained in the previous section concerning the location of the maxima, both for the single- and dual-baseline cases. For single-baseline, negligible differences appear on the retrieved vertical profiles for the $\mathrm{RVoG}$ and $\mathrm{OVoG}$ cases (extinction is slightly different). Scattering decays exponentially from the top and presents another maximum at the ground level. On the other hand, the dualbaseline profiles show slight differences, in both the filtered and not filtered versions. If we focus on the filtered profiles, two observations can be made: 1) the maximum of the volume backscattering is not located at the top neither for the RVoG nor for the OVoG, in agreement with results in Figs. 1.b and $2 ; 2$ ) the OVoG retrieved profile resembles better the behavior depicted in Figs. 1.b and 2 because the second maximum is more pronounced than in the RVoG case. Also note that although the profiles to be reconstructed correspond to the theoretical models, the best result is obtained when the filtering step is applied. This is a consequence of the original definition of the RVoG and OVoG models, which assume that the ground response is modeled as a $\delta(z)$ function and, as a result, the fitting of a polynomial profile complicates.
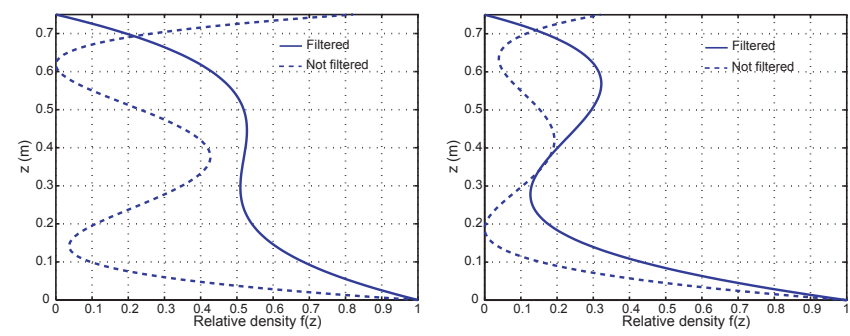

Fig. 7. Dual-baseline $x-$ pol vertical profile from the (left) RVoG and (right) $\mathrm{OVoG}$ models. Parameters: $\theta_{0}=44^{\circ}, f=7 \mathrm{GHz}, B_{1}=0.25^{\circ}$, $B_{2}=1^{\circ}, \mu=3 \mathrm{~dB}, \sigma=3 \mathrm{~dB} / \mathrm{m}$ for RVoG, $\sigma_{a}=3.5 \mathrm{~dB} / \mathrm{m}$ and $\sigma_{b}=2.75$ $\mathrm{dB} / \mathrm{m}$ for $\mathrm{OVoG}, z_{0}=-0.24 \mathrm{~m}$

\section{CONCLUSIONS}

The multi-baseline PCT approach has been tested with indoor data acquired on a rice sample. The retrieved vertical profiles do not exhibit dependence on polarization, which has been explained by the low canopy density in conjunction with the coherence sensitivity to the shape of the scattering vertical function but not to the total backscattered power.

\section{ACKNOWLEDGMENT}

The authors would like to thank S. R. Cloude for explaining some points in the implementation of the PCT. The personnel of the European Microwave Signature Laboratory (EMSL) is also acknowledged for providing the experimental data.

\section{REFERENCES}

[1] S. R. Cloude, "Polarization coherence tomography," Radio Science, Vol. 41, April 2006.

[2] S. R. Cloude, "Dual baseline coherence tomography," IEEE Geosci. Remote Sensing Letters, Vol. 4, No. 1, pp. 127-131, Jan. 2007.

[3] J. M. Lopez-Sanchez, J. D. Ballester-Berman, Y. MarquezMoreno, "Model limitations and parameter-estimation methods for agricultural applications of polarimetric SAR interferometry," IEEE Trans. Geosci. Remote Sensing, Vol. 45, No. 11, pp. 3481-3493, Nov. 2007.

[4] R. N. Treuhaft, S. N. Madsen, M. Moghaddam, and J. J. van Zyl, "Vegetation characteristics and underlying topography from interferometric radar," Radio Science, vol. 37, pp. 1449 1485, Nov. 1996.

[5] R. N. Treuhaft, and P. R. Siqueira, "The vertical structure of vegetated land surfaces from interferometric and polarimetric data," Radio Science, vol. 35, pp. 141-177, 2000.

[6] R. Treuhaft and S. R. Cloude, "The structure of oriented vegetation from polarimetric interferometry," IEEE Trans. Geosci. Remote Sensing, vol. 37, pp. 2620-2624, Sept. 1999. 